\title{
Comparing the Incomparable: 'Speaking Out' and the Use of Drawings in Notebooks by Physicists
}

\begin{abstract}
Harmen Hollestelle
CommonRoom, Bandung, West-Java 40132, Indonesia; harmhollestelle@yahoo.com

Abstract

To understand the possibility of interaction between scientific experimenting and artistic drawing in the form of drawings made in notebooks, assumed is a common element, a spiritual property, and transference as a mechanism for causality. Scientists, scientific experiments and artistic drawings all have different merits. Here the concept 'speaking out' in its meaning of expressiveness is proposed to bridge these differences. Scientific action and artistic action cannot be compared directly. However, a common spiritual element will make the investigated object, experimental set-up and notebook drawings comparable in the sense of translations authorized by the physicist. They all then speak out from the same source.

In this paper considered are recent drawings made by physicists during experimentation, in notebooks and diaries. Discussed is transferal causality between the physicist, the artistic drawing and all the relevant objects belonging to the experiment. Spiritual properties are introduced for the physicist being a person, and for the investigated object, the experimental set-up and the drawings as objects.
\end{abstract}

Keywords: physicists, experimental set-up, drawings, spirituality, interaction

\section{Introduction: Spirituality and Speaking Out}

This study aims to an understanding of the differences and similarities between human persons, objects belonging to nature such as an object under investigation during an experiment in physics, and objects created by human persons such as an experimental set up or a drawing in a notebook. Creation, here in this article, is restricted to the human context. Human persons and objects are usually regarded as having many differences. One of the differences lies in freedom. A human person can think and feel and move by him/her self and choose whereas an object cannot. I will not try now to expand this discussion towards theology. Only, to be sure, from philosophical theology one may learn that creation by humans is not the same as creation by God (Burrell, 2008). Following these theological views, only God can act as a prime creator, a 'prime mover', a human person cannot. Indeed, then, a human person is him/her self a creature, created by God. God creates out of nothing something and all that is created by God changes and varies. Human persons however can only create by changing something into something else. Human persons can act freely. They are free in choosing how to answer and act upon their feelings of responsibility and love for instance, that are part of them as human persons are created by God. Responsibility and love draw upon them and empower them at the same time. This kind of freedom clearly opposes determinism, and it opposes libertarian freedom, that does not care about responsibility. Augustine, reflecting on the creation of heaven and earth (Augustine, transl. A. C. Outler, 1955), wrote: "They (heaven and earth) cry aloud that they were made, for they change and vary. Having something not already existent is what it means to be changed (changing) and varied (varying). Heaven and earth thus speak plainly that they did not make themselves. Their voice is their visible presence." In these sentences Augustine captures what to me is the essence of spirituality. Heaven and earth cry out about their creator. Their voice is their visible presence that allows for both individual persons and singular objects to cry in their own way. The cry, the truthfully committed and desperate voice, of an object or a human person speaks out about the why of their creation, their purpose and longing.

Spirituality then means 'speaking out'. We speak out and reach others. Because our voice is linked to matter we can reach others. In what way we can speak out, what our gifts are, is concealed in the presence we have in the world. All these gifts are different, trying to compare them means comparing the incomparable.

\section{A Introduction: Describing Drawings and Displaying 'Good Behavior'}

To describe drawings with the help of quantifiable means has pro's and contra's. The emotional value of a drawing or image is difficult to capture with a number. Drawings are considered individual per se and because of this, describing them with the help of numbers is not very plausible. However the drawings considered here in this article, recent drawings made by physicists intended for notebooks, are not just drawings standing on themselves without further purpose. They are integrated parts of the notebook and thus their creation and use is 
also part of the serial of actions that lead to the final results of the experiment. Indeed, they are part of the precept for good experimentation as such. Investigating causality becomes very difficult without some means to describe these drawings objectively. Objectivity in the form of addressing numbers to drawings makes it possible to notice whether a causal stream that includes drawings is similar to another one or not.

We only consider drawings that display 'good behavior' as defined in (Ramsey, 2011). This means that a drawing with a certain kind of objective character must agree and correspond with a likewise intention of its creator at the time of creation. However we allow for the case that a drawing, when being used, causes a different or an opposite effect at a later moment. The use of drawings in physics is well investigated (Baigrie, 1996), (Hesse, 1963), (Pittman, 1999), (Woolgar and Lynch, 1990).

One manner of how numbers can be associated with line drawings or point clusters, where at the points the lines intersect. is described in (Hollestelle, 2012) to which reference is made in the following for details. In short it means the use of the notion of 'looking time'. Looking times refer to the time our attention (or better, the attention of the creator at the time of creating the drawing) needs to screen a point cluster. Looking times are well known in psychological research.

Two independent ways to estimate looking times by calculation starting from the cluster point co-ordinates on the paper leaf plane have been proposed. A kinematic approach describes screening by way of leaps from one point following the other through the whole drawing resembling a melody covering the plane. A diffusion approach describes screening by way of a small scale flow resembling a local song phrase. The kinematic type looking time is termed 'global recurrence time' (GRT) while the diffusion type looking time is termed 'local displacement time' (LDT). In the quantities calculated as estimations of looking times the factors determined by the individual person who does the screening are left out since they cancel when comparing different drawings under the same circumstances, that is, with the same person screening. In this way objective looking times are generated depending only on properties of the drawings or the point cluster.

In section 2 will be discussed the existence, within the context of an experiment in physics and depicting the experimental set-up, of two different types of drawings that can be recognized from the above looking time values. Evidence is examined for actual correspondence between these drawings appearances and the intention of the physicist, characterized as focused or non-focused, as it was at the time of the creation of the drawing. The intention of the physicist is assumed here to be his or her choice and determination to perform such and such experiment to reach a new result or to verify a result.

\section{B Introduction: Spiritual Properties and Correspondence}

In this article with spiritual will be meant: relating to the senses, as far as matter is involved, and to the free personality, as far as the immaterial is involved, these two aspects integrated to form a consistent unity. Concerning persons and objects like artworks this is a familiar concept, for objects like an experimental set-up this is new. For the physicist, intention, in the context of this article, will be argued to be a spiritual property (person wise), existing during all the time the experiment lasts and it is one that can possibly change during that time. With this I deviate, as mentioned before, from a deterministic view of persons, but instead accept freedom of mind for them.

Starting from the spiritual property intention (person wise) the spiritual properties for all the objects relevant for the experiment will be defined. They will be limited in time duration to the time span the object is created, reflected upon or actively used, in short given attention, by the experimenter. This spiritual property (object wise) corresponds at the time span or moment of creation of the object with the spiritual property intention (person wise) of the experimenter and remains always the same afterwards. It cannot change. Indeed objects, as understood here, do not possess a (freedom of) mind or a free will. Objects will include the object that is investigated, the experimental set-up, regarded as one object here for simplicity reasons, and notebook drawings. The spiritual quality of the spiritual properties (for the person as well as for the objects) depends on the attention given by the experimenter to the objects during the time the experiment lasts, while all this time the attention continuously shifts from object to object. Without attention persons and objects are isolated from each other. However the above mentioned correspondence is taken to hold only at any time of creation.

What more precisely is meant with correspondence is discussed ultimately in the final section. It is clear that equality is not a useful concept here because for instance the spiritual property intention (person wise) can change and the spiritual property (object wise) cannot.

The investigated object is a special one because it already existed before the time of the experiment. Mostly it is an object from nature and it is not created by the physicist within the experiment like the other objects are. Still there is a creative moment. Correspondence between the spiritual property (object wise) and the experimenters intention (person wise) is now assumed to hold during the time creative reflection leads to new expectations, for instance expectance of a new theory that differs from former ones. Correspondence would be useless when it would hold otherwise, since then at that time the intention (person wise) of the physicist would agree with 
former results and former theories and no new experiment would be initiated. From this consideration one already notices the difficulty of defining the concept correspondence or even only to give a user 'manual' because it deals at the same time with persons and with objects that all have a different character.

\section{C Introduction: Equivalence and Transferal Causality}

Instead of regarding the causal stream, in terms of transferal causality: the flow, existing or flowing between material objects, where physicist and relevant objects are all regarded as material objects, like in a materialistic view might be possible, now both the physicist (as a person) and the investigated object, the drawing and the experimental set-up (as objects) can possess a spiritual property (person wise or object wise) and this quantity (person wise) in this case can only remain to the one physicist (because as experimenter we understand in this article always only one single person) while this quantity (object wise) flows between any of the objects given attention by the physicist one after another. For simplicity reasons only the time duration of one experiment is considered here, not described is a larger time span or when also more physicists might be involved.

The causal flow (object wise) from object to object can only be traced when in some way the involved objects still have something in common. The objects occurring in an experiment however all have a different character. To identify a common element among them the concept equivalence is used. Equivalence is defined to exist between objects when one is considered to be a translation of the other. A translation emerges when during the creation of an object the experimenter expresses, 'speaks out', through the attention given to the object, of his/her original spiritual property intention (person wise). Thus a translation depends for authorization on the experimenter, starting from the investigated object. Once objects are created, spiritual properties (object wise) do not change and only exist when the relevant objects receive attention one after the other, the objects being translations for that time. Here the spiritual character of the causal flow becomes clear. The common element within the translation of for instance words does not only depend on the involved word sounds, but also on the word content, a combination that is bound to be spiritual. The time-order of the attention change, attention given first to one object and then to another one, determines the causal order and causal flow (object wise).

In the final section is discussed how causality is possible in the here studied case of different fields of action as drawing and physics are. In this description I aim to do justice to the different character of persons and of all the kinds of objects involved in an experiment in physics. This is important, apart for its own sake, also because necessarily any generalizing description, like for instance a materialistic one, will tend to rule out differences. While this article sets out to prepare a description of causality suitable to be used when including drawing in the context of a scientific experiment, I have to consider persons: the physicist, and objects from nature: the investigated object, and created objects: notebook drawings and the scientific experimental set-up, with respect to their interaction. To compare the persons with the objects the concept correspondence is introduced, to compare objects with each other the concept equivalence is introduced. This consideration is fruitful because, thanks to the spiritual (material and immaterial) status of art-works like drawings, it brings to the foreground the possibility of the introduction of spiritual properties into causality.

\section{The Experimenters Intention as Focused or Non-Focused: Examples of Correspondence with Drawings in Notebooks}

The intention of an experimenter, and in this article I mean a physicist who also creates a drawing in a notebook, can be described to belong to two different categories. There are the researchers that intend to survey a large collection of possible results from a large field that has to be examined, often called field work. And on the contrary there are the researchers that intend only for a few, say two, possible results from their experiment, often called yes/no experiments. The field work experimenters intention can be termed to be non-focused and the yes/no experimenters intention to be focused.

Can these two intention categories be recognized in possible types of notebook drawings? And, can these drawing types then be characterized by number groups? It turns out (Hollestelle, 2012) that the looking times in terms of numbers that belong to individual drawings can be used to separate and recognize two independent types of drawings grouped according to low LDT number or high GRT number. The first type of drawings was found then to correspond with focused intention of the physicist and the second type of drawings to correspond with non-focused intention. Displaying 'good behavior' comes out to be very important here. Without this the above correspondence would be lost.

What do drawings look like when they belong to one of these two types? At the end of this section two examples of notebook drawings are shown, figure 1 and figure 2, taken from the collection of drawings included in the above reference. They describe the performance of the same experiment but show completely different qualities. 
Figure 1 depicts many details that are scattered over the field of the drawing independent of whether the details are decisive for the result or not. The other drawing, figure 2, depicts only the essentials of the experiment, meaning that part of the experiment where the decision is made what the result will be: (in this case) does or does not there occur interference of rays.

Both experimenters that made these drawings performed the experiment equally well. Where they differ, this is because of their intentions. While they value different aspects differently, this does not mean that the two intentions should be valued differently in terms of good or bad experimentation. One intention is non-focused and the experimenter is from the beginning open to deviating results that do not necessarily answer the question that determined the experimental set-up. The other intention is focused and this experimenter is determined not to be distracted from finding the yes or no result about the experimental question. These characteristics can be re-found directly in the appearances of the drawings the two experimenters made, figure 1 and figure 2 respectively.

The situation that emerges from the investigation of all the drawings in the collection is such that one may name and classify, in correspondence with their experimenters/creators intention, certain drawings as focused and other drawings as non-focused. 'Good behavior' is the essence of correspondence. Assuming it seems natural and necessary if one wants to believe in scientists and science. The absence of 'good behavior' would be difficult to imagine when one accepts consistent unity of spirituality aspects, also in the light of the explanatory discussion section at the end of this article.

Een tekening van de opstelling:

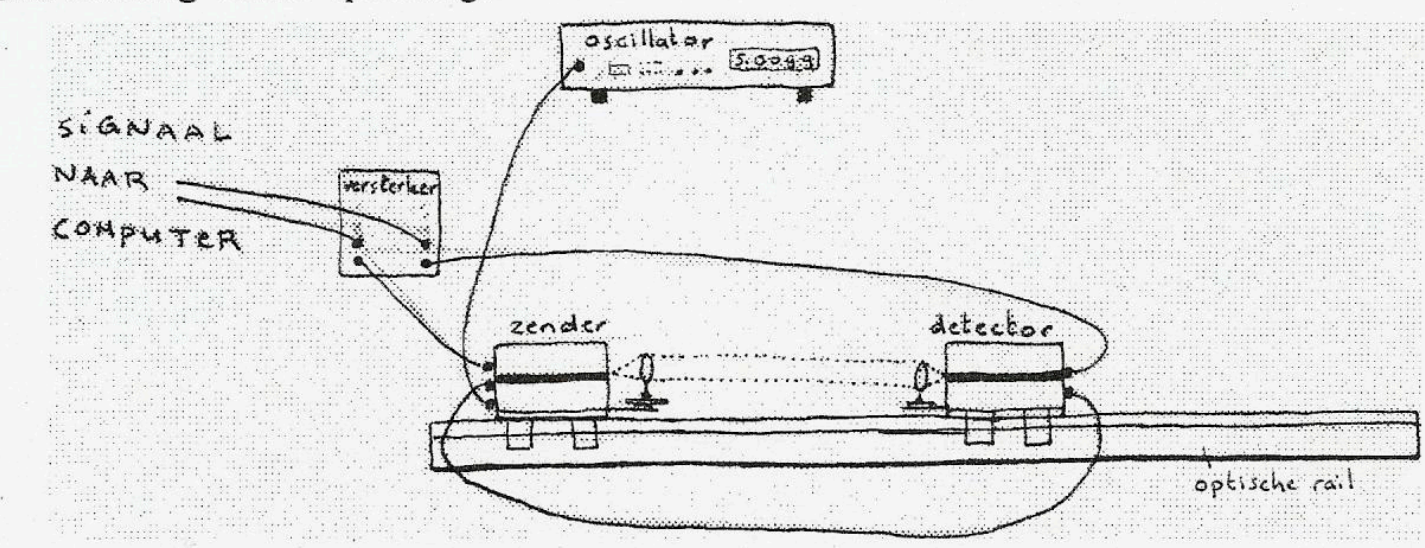

Figure 1 


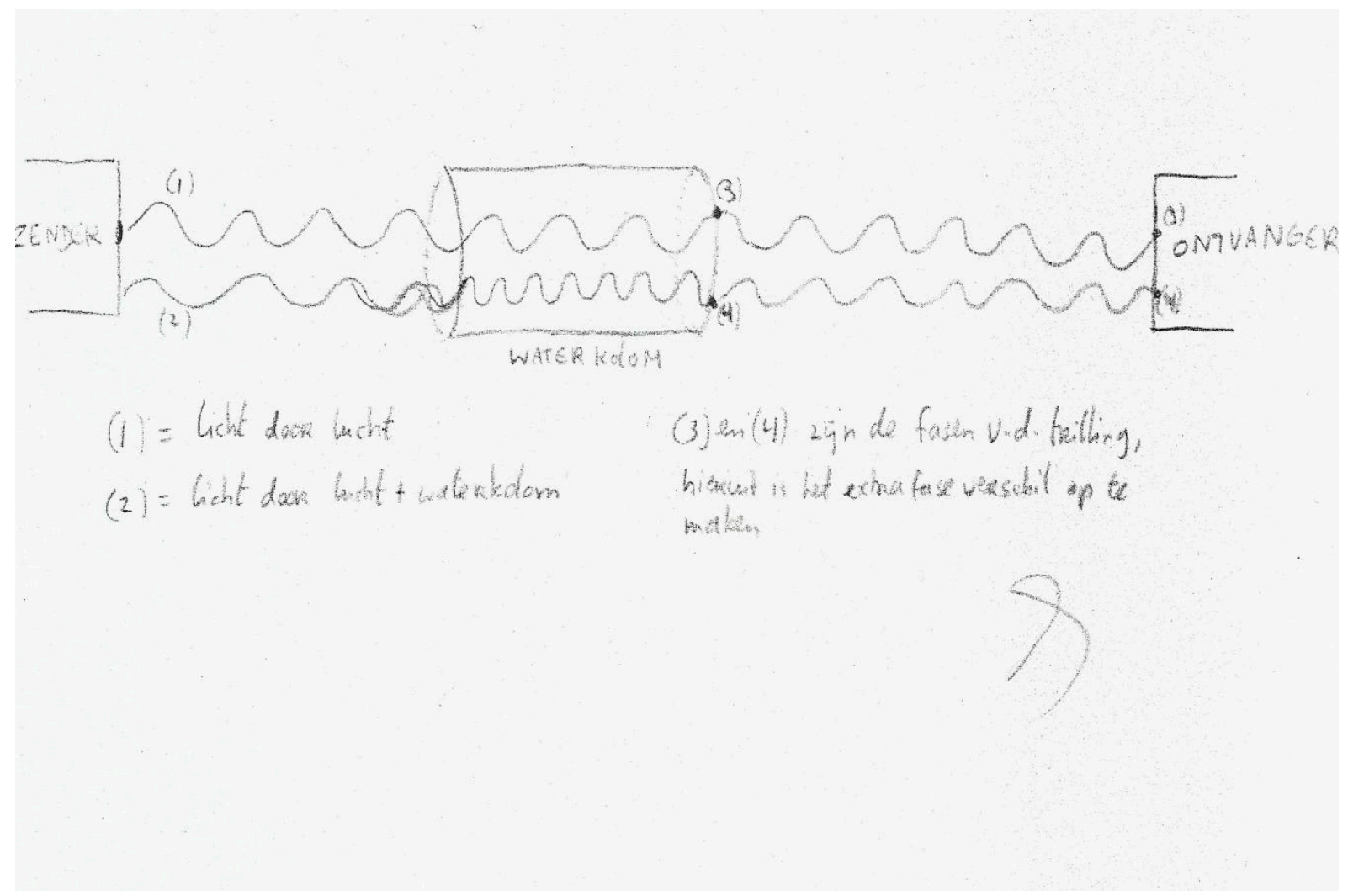

Figure 2

\section{Drawings and the Timeline of an Experiment}

One may differentiate generally between two kinds of timelines when a drawing is part of an experiment. The experimenter can create and use the drawing at two different moments in the scheme of experimentation. One scheme is as follows. One starts with deciding on the initial intention (to perform such and such experiment to reach a new or verify such and such result) by for instance reflecting on earlier results such as at that time known properties of the object under study. Then after this follows the creation and use of the experimental set-up together with at the same time creating and using a drawing depicting this experimental set-up, and finally a new result is reached or a result verified. Another scheme is this: again one starts by reflection upon the results of other earlier experiments, when deciding on the initial experimental intention, however while now at the same time creating a drawing depicting the newly imagined set-up, after this followed by the creation and use of the experimental set-up and finally reaching or verifying results.

Most other places of creating and using a drawing in the stream of actions can be reduced to these two schemes. Due to the places in the stream, the experimenters initial intention will influence later on the creation and use of the drawing that itself will accommodate directly to the creation and use of the experimental set-up (scheme 1), or the experimenters initial intention and the creation of the drawing accommodate directly and both will influence later on the creation and use of the experimental set-up (scheme 2). In both schemes the drawing is understood always to display 'good behavior' during the time of its creation.

Accommodation is possible because deciding upon the initial intention takes time, and so do the creation of the experimental set-up and the creation of the drawing. Accommodation is a process where two (or possibly more) activities are performed in overlapping time spans. During the time they are both performed simultaneously the activities directly influence each other and merge so that in the end one may speak of them as having one single connected content. Accommodation is the process at work towards correspondence. When influencing-later-on, one activity is performed earlier than the other without overlapping time span and there will be causal order. The notion included in transferal causality: effects are later in time then its causes, has been discussed extensively in the literature (Mackie, 1974), (Tang, 2011). One of the examples to prove it wrong has been repeated many times. It originates from Kant (Kant, re: 1956): consider a heavy sphere resting on a cushion that gives way under its weight. Since the sphere and the cushion are at rest one assumes that cause and effect here are simultaneous. However when one considers the way the above sentence formulates the situation one realizes and sees as if in slow motion that the cushion gives way to the sphere only after the sphere is put down on top of 
it by us, when the sphere is left to its own after our action. Putting the sphere on it causes the cushion to give way just after that. In this way one understands that this situation does not display simultaneous causation. I will not however in this article elaborate on this further.

\section{Drawings, Properties, Numbers and Theories}

Within the description of transferal causality a conserved quantity is transferred from the cause to the effect, the effect assumed to be later than the cause. Definitions and arguments for (and also against) transferal causality in general are given in for instance (Dieks, 1986), (Kistler, 1998). The introduction of spiritual properties allows one to understand causality as transferal, including the transfer of a conserved quantity that is rewritten in terms of the transfer of a conserved spiritual property, (person wise) between the persons involved (only the one physicist here however) and (object wise) between the investigated object, the notebook drawing and the experimental set-up according to the attention given to them. Since the experimenter gives attention one by one to the objects continuously, transfer (object wise) follows simply the timeline of the experiment. The transfer (object wise) is achieved through the person (the physicist) who creates, reflects upon and/or uses all the relevant objects. What can be a conserved quantity is defined within physics usually. Conserved quantities are ontologically dependent since they are properties, while within the description of transferal causality there is imagined to be a 'flow' of this quantity from the cause to the effect.

For the spiritual property (person wise) is chosen the physicists intention, an intention that possibly could change in time depending on the moment on the timeline. There is the initial intention of the physicist when before performing the experiment reflecting upon the investigated object, and there is the intention of the physicist as the creator and user of the drawing, and there is the intention of the physicist as the creator and user of the experimental set-up. These intentions all can be different and vary in time in principal. A similar spiritual property (object wise) of any relevant object, also called intention (but now object wise), will directly depend on the physicists intention as spiritual property (person wise), that is, just at that time that the object is given attention while it is created. We may assume, following the two above discussed timeline schemes of experimentation, a relation to exist, according to accommodation or influencing-later-on, between the above intentions (person wise) at different times on the timeline. Flow and conservation of the spiritual property (person wise and object wise) follows from this relation as will be shown.

We will now discuss the existence of conserved quantities and flow in this new respect of spiritual properties. First it will be argued that properties (of drawings) and the looking time values describing them, as introduced above, can be used simultaneously and consistently together. This is necessary because the values are used to classify these properties. Then the relations between properties and relevant theories are discussed where both should have the same ontological status, i.e. object dependent, to avoid inconsistency within the history of these theories. After this in the following sections the introduction of the spiritual properties is possible and a conserved flow can be understood in terms of these spiritual properties, within the realm of experiments and notebook drawings by physicists.

There is reciprocity between the properties of an object and its description in terms of numbers. Extension of an object can be described with the numbers that relate to its spatial shape. Concepts like 'belong to' and 'being connected to' find their counterpart in these numbers. Naively the shape of an object is reflected in the symmetries and similarities between the describing numbers. What is 'inside' the object is reflected for each direction by an interval, a series of numbers that all are below or equal to a certain value that represents the surface. With surface one may mean that part of the object that is sensible to touching by another object without changing the object's shape.

Measuring then is continuing until. When relating to a physical reality, like a part of space or an extended object in space, the numbers that are the result of measuring, of measurements, are properties of this reality.

Measurement of extension is continuing in one direction, starting from some chosen constant point at the object, until one stops continuing at the surface. For a certain type of measure to have meaning, for instance when one measures something to be say 'two', this implies that measuring 'one' should have a meaning to start with. Counting may have several different appearances (Murawski, 2010): counting may mean counting again and again the same event (say 'heartbeat'). Or it may mean counting along a scheme, resulting in the number 'one' as singularity, 'two' as spin, etc. Then there is counting like I want to discuss it here, namely counting as comparing, due to Euklid (Euklid, transl. C. Thaer, 1991): defining as 'one' a certain measure that is the property of a certain object and then comparing the next object and a related measure with it. In this last instance counting is comparing 'one' with the notion: another 'one'. This other 'one' can be larger than or smaller than the original 'one' or without difference. Generally one is interested in what then the next number is starting with a certain given original number. The next number follows on the original number, ideally with only a 'one' as difference. 
It is the possibility of noticing change and difference that makes comparing possible and makes the existence of successive numbers possible. In this description numbers are ontologically dependent, a proof of ontological dependence of numbers is given in (Schwartzkopff, 2011). Thus the reciprocity between the properties of objects, being themselves ontologically dependent on the objects, and their description in terms of numbers is consistent.

Of course the definition of the meaning of any property, and its measurement, is theory laden. One cannot speak of, or measure, the weight as a property of an object, for instance, without implicitly referring to some theory of gravitation. One could, as a simple example, argue, as has been done in the past, that the weight of an object has to be regarded as its tendency to fall to the earth surface (Dijksterhuis, 2000). In that case for the theory to relate somehow to reality there should be available a collection of experiences from the past where the relation between weight and this tendency was established. To avoid circularity, in general there have to be causal relations within historical series of theories and experiences where new theories and new experiences follow upon each other in time and influence each other. Also all the time theory and experiment in one direction of research are given input from outside by new ideas and theories from other disciplines and by independent technical innovation, discoveries and creations. What emerges is that a property of an object and the related theory are both ontologically dependent on the object. Property and theory are discovered from the just before that time available properties and theories (available theory and properties at some time do not have to agree). For arguments for and against regarding theories as being discovered contrary to being created, see for instance (French and Vickers, 2011). A full description of a view where theories describe objects while they themselves resemble properties of these objects will be developed in a forthcoming article.

When a theory is called false, i.e. not agreeing anymore with the available properties of that time, this only means that the relevant collection of experiences from the past it refers to is outdated and too small. Thus, with the theory that the earth is flat comes a large collection of short distance experiences but missing is the experience of travelling around the world. When expanding the relevant collection in this case, this leads to discovery of the theory that the earth is round. However one can still speak of the outdated theory as discovered and true within its limits, at short distances at earth, remaining ontologically dependent on the object earth.

The interpretation of drawings made by physicists in terms of two types starts with determining the co-ordinates of the intersections of the lines the drawings exist of. From a kinematic and a diffusion model one derives algorithms that calculate values, the 'looking times', for each drawing. These values, numbers, can be divided into two classes, from which one recognizes the existence of the two types of drawings, that turn out to correspond with the pre-existent person wise intention types yes/no experiments and fieldwork. The framework here is the pre-existence of earlier experiments and their description in terms of 'yes/no' experiments or in terms of 'fieldwork'. The interpretation of drawings belonging to these two types is already precluded by assumptions of the relevant community, here the community of physicists (Hesse, 1974). To which type an individual drawing belongs depends on 'good behavior' during the creation of the drawing and on the intention (person wise) of the individual physicist.

\section{Spiritual Properties Person Wise: Giving Attention and Speaking Out}

As spiritual property (person wise) chosen is the intention (person wise) of a person, and this spiritual property will be examined first. Can the intention (person wise) of a person fulfill the requirements for a spiritual property like they are described in the introduction? In this article the person will be the physicist initiating and performing an experiment and creating and using a drawing in a notebook depicting the experimental set-up like was described in section 2. Intention (person wise) was there for simplicity reasons classified in two types, focused and non-focused. Intention (person wise) of the physicist is necessarily accompanied by his/her attention to the outer world that consists here of the investigated object, the experimental set-up and of the notebook drawing. For simplicity I leave out other persons like colleague physicists, since I assume that the experiment is performed by only this one person.

For our description of intention (person wise) accompanied by attention to be consistent we need to check whether the two above intention types are supported by similar attention types. Concentrating for now on the creation of drawings one may describe giving attention, called screening in section 1A, in two different ways, using the approaches introduced before: a kinematic model approach and a diffusion model approach. There are: the point cluster a drawing exists of, the attention type and the intention type (person wise).

We now work out the inter-dependence between a drawing and creative attention, the attention at the time of creation of the drawing, as the directing guide on the paper leaf. A cluster with approximate translational symmetry in any direction, like a melody or cloud, agrees with a long term creative attention of the artist, working along all parts of the drawing again and again without center, say: like how making a knot looks at a 
distance. A short term creative attention of the artist agrees with a different kind of cluster shape. This attention type may single out one direction as specific, say: like how a wave looks at a distance, agreeing with a cluster with translational symmetry in one direction, like a local song phrase.

Re-called is now the correspondence between a drawing and the intention of the creating physicist. The descriptions of creative attention and intention (person wise) of the physicist appear then to be consistent: the property intention (person wise) (non-focused) turns out to be accompanied by a melody-like drawing and a knot-like creative attention, and the property intention (person wise) (focused) turns out to be accompanied by a song phrase-like drawing and a wave-like creative attention. After extrapolating this consistency relation between intention (person wise) and attention to all the time of the experiment and including as objects given attention not only the drawing but also the investigated object and the experimental set-up, indeed with intention (person wise) we have a good candidate for a spiritual property of the physicist.

Special care is needed for the treatment of the creative attention given to the investigated object. Intention (person wise) and creative attention now co-operate by creative reflection. The freedom of mind of the physicist clears the way for the possibility that his/her intention (person wise) at that time will not correspond anymore completely with former properties and former theories of the already present investigated object. Creative attention is now a guide away from known properties towards newly expected ones. In this way the physicist is able to intend a new experiment depending on for instance the expectation of a new theory, as a property of the investigated object, that has to be verified by this new experiment. Created is an experimental idea and a commitment, the investigated object is 'turned around', looked at from a different angle and changed thereby into a different position in a new light.

Spiritual properties should, like was mentioned in the introduction, involve both the material and the immaterial. The property intention (person wise) of the experimenter clearly fulfills this requirement: the personality of the physicist and the space/time material and bodily aspects of the physicist and of all the relevant objects given attention initially within the experiment together make up for the complete spiritual quality of intention (person wise). By giving creative attention to an object the physicist 'speaks out' of his intention (person wise) towards the object, molding the object towards its use within the experiment. 'Speaking out' is not to be meant verbally but is to be interpreted as shorthand for a complete notion of 'expressing yourself'. Also the property intention (person wise) will remain existent during the whole timeline of the experiment while attention shifts from object to object. Intention (person wise) can change in time, if only because any person is free to change his/her mind.

\section{Spiritual Properties Object Wise: Speaking Out Object Wise}

We now likewise define intention (object wise) as a spiritual property for each of the relevant objects of the experiment, as corresponding to the intention (person wise) of the physicist during the time of creation of the object (in the case of the investigated object: its time of creative reflection). Correspondence at the time of creation means that intention (object wise) in return, at the time of creation but also afterwards when it is given attention, will 'speak out' from the objects matter and form about the how and why of the experiment as it is intended (person wise) by the physicist just at the time of creation of the object when the physicist 'speaks out' towards the object. Examples of correspondence were already given in section 2. This implicit definition of intention (object wise) is as close as one can get to it as objects are assumed not to possess freedom or a free will. Speaking out (object wise) in this context is depending on attention given by a person, an object cannot have the initiative, or itself give attention, and the relation by 'speaking out' is not symmetrical. Also because of the objects lack of freedom its intention (object wise) cannot change anymore after its time of creation, not considering the possibility that the object is changed into something else by outside interference.

The spiritual property intention (object wise) belongs to the object only during the time of its creation, reflection or use. Thus an object possesses the spiritual property intention (object wise) in any way only during the time span the object is actively a part of the stream of actions of the experiment. Outside that time span, another object that is now at that other moment being created, reflected upon and/or used and now, like the former object was before, actively part of the same stream of actions, will possess its own intention (object wise), depending on the physicist speaking out to it at its own time of creation. Although not quite usual for an investigated object or experimental set-up in physics, it is common to speak of spiritual aspects of artworks in this way, so this definition should not sound improbable. Special here is the time-limited character of the spiritual property (object wise), limited to the time of creation, reflection or active use of the object only.

With this definition both the material and immaterial involvement of the spiritual property (object wise) is secured. Indeed intention (object wise) as property of an object involves the material of the object and its possibilities and restrictions at the time of creation, or creative reflection, and it involves the personality of the experimenter and his/hers choices, longings and goals as they are just at that time. 
We are allowed to infer that intention (person wise and object wise) is a conserved quantity in the large: when changing attention between any of the objects the physicist remains generally speaking true to the initial intention (person wise). However the physicists attention changes, while the intentions (object wise) follow upon each other in time. These will then be called equivalent: they differ only because for instance the objects drawing and experimental set-up belong to different fields: contingent art and demonstrable science. Existence of equivalence will be argued below. Equivalence between intentions (object wise) as spiritual properties of objects of different fields is now considered sufficient for regarding the flow of intention (object wise) between these objects, that have a certain time-order for their moments of creation, reflection and use following the timeline schemes discussed above, conserved.

For the immaterial involvement of the intentions (object wise) existence of equivalence seems logical because this involvement depends on the intention (person wise) of the physicist, the very same person for the investigated object, the drawing and the experimental set-up, who is considered to remain true to the initial intention (person wise) at the start of the experiment. All relevant objects will 'speak out' of their own special contribution, their gift, to the same experiment, depending for their specialism on their specific material form, but in general confirming the original purpose of the experiment. For the material involvement of the intentions (object wise) one has to consider that the objects are created, or creatively reflected upon, within a certain spatial order that is the same for all because it depends on the material (body), mind, sensibility and senses, and choices of the same person. Therefore, for the objects, the material involvement of the property intention (object wise) will display itself, will 'speak out', although through different material, as this same spatial order. Spatial order is taken broadly: the material, mind, senses, sensibility and choices of the physicist are decisive for the choice of material and decisive for the form, the spatial order, this material will get within his/her hands. This means the existence of equivalence in this respect too.

Equivalence is not the same as equality since the objects it concerns belong to different fields and equality is not possible. To compare the intentions (object wise) one needs a less strict and more versatile concept, like equivalence is, that depends on a kind of 'authorization' by the physicist. Equivalence then resembles faithful translation. Notice that translation has a specific time order, from original to translation, just like causality should have from cause to the effect. Indeed as an example of equivalence for instance an experimental set-up in all its three-dimensional material aspects can be imagined to admit the same kind of spatial order description as are possible for two-dimensional drawings as described above. This should be so because otherwise no drawing could exist, related to the experimental set-up, depicting it faithfully in all its relevant experimental aspects. And such drawings do exist and fulfill this requirement of faithfulness, as is confirmed by the collection of drawings of experimental set-ups already mentioned in section 2 .

During the time of the experiment and while attention changes from object to object many influences from outside the experiment will be endured. There is a chance that the physicists intention (person wise) and his/her attention will be influenced too and part of the intention (object wise) will be 'dissipated' and 'lost' to the surroundings during this process. Any kind of distraction or noise can exert such influence. Of course this dissipation is not a real loss, only a kind of diverted flow of intention (object wise) towards objects belonging to the outside surroundings instead of to the experiment. The physicist stumbles so to speak over some outside object of which the existence was not realized before. An outside object might involve outside persons and the intentions (person wise) of the physicist and of the outside persons in that case can interact. In this article this interaction is not further described.

During their time of creation and use, a drawing or the experimental set-up can make the experimenters initial intention (person wise) as it was at the start of the experiment change too: the experimenters attention can be directed towards space/time aspects not considered before in relation with the experiment for instance. Still the experiment and its performance remain inside the realm of physics. This then should not be understood as a diverted flow of intention (object wise) to outside the experiment, but as a diverted flow of intention (object wise) within the experiment. Space/time aspects as they are meant here, whether drawing-related or experimental set-up-related, are considered to be among the basic aspects that are always part of any experiment in physics. The spiritual properties, for the physicist and for the relevant objects, are not yet defined outside the timeline of a single experiment. Here the history the experiment belongs to will help. For simplicity reasons I will not in this article further outline the continuation of definitions.

What remains is the existence of a flow of the spiritual property intention, conserved in principle in the large but tolerating diverted flow, object wise between investigated object, the drawing and the experimental set-up on the one hand and person wise restricted however to the single one physicist on the other hand, when these are related to each other in the in this article meant way. 


\section{Discussion: Comparing the Incomparable}

In this article discussed is thus far that drawing, and here I mean drawing restricted to notebook drawing by a physicist during experimentation, can be part of the performance of the experiment in a intelligible way. This is only a sketch of their interaction starting from some elementary assumptions. The difficulties engaged in dealing with persons and different kinds of objects maybe do not resonate through in the application of the words creation, reflection, or use, to all objects. The consideration that persons, and objects belonging to nature, the arts, and science respectively, possibly interact impels one to verify somehow the meaning and existence of their relations, as is done here with the help of the above introduced concepts correspondence and equivalence. Where there is interaction there is openness. When drawings are involved, correspondence and equivalence depend on 'good behavior' and on faithfulness. More broadly I use the notion 'speaking out' to denote interaction. Here it is the spiritual property that persons and objects have in common in some sense at some times. Understanding causality, in a transferal way, in the above description depends on this. Cause and effect can be discriminated following the timeline of the experiment when interpreting the transfer of the spiritual property (object wise) as a translation from one object field to the next one, authorized by the physicist.

Above we saw that a change of the experimenters focus type corresponds with a change of drawing type. But how can this interaction actually be possible. Drawing clearly belongs to a different field of action: contingent art, than experimentation: demonstrable science.

When one considers the specific art form 'land art' this illuminates how interaction can be possible. Land art contemplates the relation nature - humankind, often in the form sky - humankind or sky - earth. Land art type drawing is an action that uses extension and time in a fundamental way and has space, spatial extension, and time, duration, as its objects that are very much similar to the basic and ultimate objects of experimentation in physics. But object-similarity is not all there is to it. The focus type in land art just as in notebook drawings depends on the type of contemplating the artist prefers or needs. Contemplating is like giving attention, only the word expresses more easily and better how mental activity depends on the body. Creating a drawing both as part of land art or in a notebook thus also involves the personal make-up and body of the physicist, that co-determine any of his/her choices possible. Besides recognizing the mind-set and goals during an experiment by faithfully depicting the experimental set-up, creating a notebook drawing then makes it possible for the physicist to reflect, perhaps without consciously striving to this, on his/her performing and motoric skills and on the chosen material of the experimental set-up, that are just as well related to space and time, however in a different, most sensitive, way. It is a moment of relaxation and of a different kind of reflection, that of drawing.

Artistic research is the name one frequently finds in the literature for art as research, with which one means art that includes some sort of reflection, beyond simply depicting or copying a part of reality (Borgdorff, 2012). Artistic research like this is assumed to have a 'boundary object' as interest, while the artistic research itself is called 'boundary work'. The meaning of one and the same boundary object differs along with the kind of research environment, artistic or scientific. Thus a boundary object is then called pre-defined, giving rise to differing research efforts and lacking ontological content. However I feel at unease with notions where an object, real or abstract, depends on and varies completely, for its meaning and content and properties, with its environment. I like to hold on to a distinction between art and science in the way of aesthetic appreciation and empirical verification respectively, assuming one and the same object. While empirical verification may be a clear concept to most scientists, aesthetic appreciation in this context is not so clearly defined. With it I do not mean a general style or fashion, for instance in physical reasoning, like is advocated for instance by James McAllister (McAllister, 1996). For me the aesthetic is individually appreciated, while truth appears to the individual as an image from the object under consideration. Summarizing parts of play writer Harold Pinter's Nobel Prize lecture (Pinter, 2005): "The search for truth is clearly what drives the endeavor. Often you stumble upon the truth without realizing that you have done so. But there are many truths. In a sense the author is not welcomed by the characters. They resist him, they are impossible to define. But finally you find that you have real people with will and sensibility on your hands, while you are unable to change them, to manipulate or distort them." This view corresponds very well with the one of painter Jackson Pollock, whose action painting involves getting into a relation with the painting, resembling a giving and taking (Emmerling, 2004).

Just giving names is not yet sufficient to bring an explanation to the original question, how is interaction between different fields of action possible?. In this article I try to find the common ingredient, where do the above research environments overlap, what is their boundary then? For drawings made by physicists in notebooks an answer may be found in that one action of the human body that cannot be circumvented in art, and not in science too surprisingly, breathing.

When initiating an experiment in physics one has to feel and think about the object and the way to reach its properties with fresh eyes, opening upon it a new window, to be able to perform an original experiment. Similarly the encounter with the emptiness of the new leaf that is used for drawing enables one to start feeling 
and thinking from scratch. To quote John Blockley, writing on how to learn water-color painting: "The greatest difficulty is ... in achieving new ways of looking and thinking. Inexperienced painters ... strive to duplicate accurately and copy in everyday acceptable terms. Their main problem is in adjusting to a different, intensely visual way of looking. Painting is about freedom of everyday labels. Freed from our conventional associations, we really begin to look and observe. We look for color, shape, patterns, the relationships of one 'thing' to another, find the ... intrinsic value of things. We start to select, and paint those parts which interest us. " And: "Brush and color movements are the vocabulary of water-color and should be instinctive, leaving the mind free to concentrate on the idea that prompted the painting." (John Blockley, 1985)

Thus there is through both fields of action an opportunity for a new start, proverbially opening up the spirit and letting one breathe freely. Spirit and breathing are two sides of the same uniting aspect: they unite the bodily and motoric kinematic space time senses, and the mind and the personality from the time when breathing started onwards. Together spirit and breathing make 'speaking out' possible.

Let me read a passage from Kilwardby (Fidora, 2011) who is discussing the differences between contingent arts and demonstrable science in the thirteenth century:

“... since the diversity of the subject matter, namely of the necessary or contingent terms and assertions, does not introduce a diversity among that which constitutes the object of science and that which constitutes the object of opinion."

Demonstrable and contingent types of knowledge differ not because of the object one is dealing with, nature, but because of the subject. Before a bird's eye view there is no difference. Thus causality in both physics and the arts can be treated at one time.

\section{References}

Augustine, (transl. A. C. Outler), 1955. Confessions, Bk. 11, Chap. 4, (http://www.ourladyswarriors.org/saints/augcon11.htm\#chap4)

Baigrie B., (Editor), 1996. Picturing Knowledge, University of Toronto Press

Blockley J., 1985. Watercolour, Practice and Progress, A \& C Black, London

Borgdorff H., 2012. The Conflict of the Faculties, Leiden University Press

Burrell D. B., 2008. "Creator/Creature Relation: The Distinction vs. Onto-Theology", Faith and Philosophy, 25:2

Dieks D., 1986." Physics and the Direction of Causation", Erkenntnis, 25: 85

Dijksterhuis E. J., 2000. De Mechanisering van het Wereldbeeld, Meulenhoff Amsterdam

Emmerling L., 2004. Pollock 1912-1956, Taschen Verlag

Euklid, (transl. C. Thaer), 1991. Die Elemente, Buch VII, W.B. Darmstadt, pp.141: "Einheit is das, wonach jedes Ding eines genannt wird" and "Zahl ist die aus Einheiten zusammengesetzte Menge".

Fidora A., 2011. "Causality, Contingency and Science in Robert Kilwardby", Annuario Filosofico, 44:1

French S., P. Vickers, 2011. “Are There No Things That Are Scientific Theories?”, BJPS, 62: 771

Hesse M., 1963. Models and Analogies in Science, Newman History and Philosophy of Science Series 14, Sheed and Ward, London

Hesse M., 1974. The Structure of Scientific Inference, Macmillan Press

Hollestelle H., 2012. Manual for drawings: Experiment and its visual counterpart, auto-edition, Amsterdam (Chap. 1:

arXiv:0908.3902VI, Chap. 2: arXiv:1007.2844VI, Chap. 3: arXiv:1011.6300VI)

Kant I., (re-edited), 1956. Kritik der Reinen Vernunft, A203, F. Meiner Verlag, pp.253

Kistler M., 1998. "Reducing Causality to Transmission", Erkenntnis, 48: 1

Mackie J.L.,1974. The Cement of the Universe, A Study of Causation, Clarendon

McAllister, J. W., 1996. Beauty and Revolution in Science, Cornell U.P.

Murawski R., 2010.” From the History of the Concept of Number", Poznan Studies, 98: 95

Pinter H., 2005. Nobel Prize for Literature Lecture, The Nobel Foundation, Sweden

Pittman K., 1999. "Student-generated Analogies, Another Way of Knowing”, J. Res. Sci. Teach., 3: 1

Ramsey W., 2011. "Representation Reconsidered”, (review Mark Sprevak), BJPS, 62:3

Schwartzkopff R., 2011. "Numbers as Ontologically Dependent Objects: Hume's Principle Revisited", Grazer

Philosophische Studien, 82: 353

Tang Z., 2011. Causal Asymmetry \& the Explanatory Constraint, Cranmore

Woolgar M., S. Lynch, (Editors), 1990. Representation in Scientific Practice, MIT Press

(C) 2016 by the authors; licensee Preprints, Basel, Switzerland. This article is an open access article distributed under the terms and conditions of the Creative Commons by Attribution (CC-BY) license (http://creativecommons.org/licenses/by/4.0/). 